\title{
Properties of $\lambda$-Pseudo-Starlike Functions of Complex Order Defined by Subordination
}

\author{
Kadhavoor R. Karthikeyan ${ }^{1,+} \mathbb{D}$, Gangadharan Murugusundaramoorthy ${ }^{2,+} \mathbb{D}$ and Teodor Bulboacă ${ }^{3, *,+}$ (D) \\ 1 Department of Applied Mathematics and Science, National University of Science \& Technology (By Merger of \\ Caledonian College of Engineering and Oman Medical College), Seeb-111, Muscat P.O. Box 2322, Oman; \\ karthikeyan@nu.edu.om \\ 2 Department of Mathematics, School of Advanced Sciences, Vellore Institute of Technology, \\ Deemed to Be University, Vellore, Tamil Nadu 632014, Tamilnadu, India; gms@vit.ac.in \\ 3 Faculty of Mathematics and Computer Science, Babeş-Bolyai University, 400084 Cluj-Napoca, Romania \\ * Correspondence: bulboaca@math.ubbcluj.ro; Tel.: +40-729-087-153 \\ + These authors contributed equally to this work.
}

check for

updates

Citation: Karthikeyan, K.R.; Murugusundaramoorthy, G.;

Bulboacă, T. Properties of

$\lambda$-Pseudo-Starlike Functions of

Complex Order Defined by

Subordination. Axioms 2021, 10, 86.

https://doi.org/10.3390/

axioms10020086

Academic Editors: Andriy Bandura and Silvestru Sever Dragomir

Received: 8 March 2021

Accepted: 3 May 2021

Published: 7 May 2021

Publisher's Note: MDPI stays neutral with regard to jurisdictional claims in published maps and institutional affiliations.

\begin{abstract}
In this paper, we defined a new class of $\lambda$-pseudo-Bazilevič functions of complex order using subordination. Various classes of analytic functions that map unit discs onto a conic domain and some classes of special functions were studied in dual. Some subordination results, inequalities for the initial Taylor-Maclaurin coefficients and the unified solution of the Fekete-Szegő problem for subclasses of analytic functions related to various conic regions, are our main results. Our main results have many applications which are presented in the form of corollaries.
\end{abstract}

Keywords: analytic functions; Bazilevič functions; Jackson's $q$-derivative operator; starlike and convex functions; subordination; Fekete-Szegő problem; coefficient inequalities; $q$-calculus; error function

MSC: $30 \mathrm{C} 45 ; 30 \mathrm{C} 80$

\section{Introduction}

Bazilevič [1] introduced the class $\mathcal{B}(t, \zeta, g)$ of functions which is defined by the integral:

$$
f(z)=\left\{\frac{t}{1+\zeta^{2}} \int_{0}^{z}(p(\eta)-i \zeta) \eta^{-\left(1+\frac{i t \zeta}{1+\zeta^{2}}\right)} \eta^{\frac{t}{1+\zeta^{2}}}[g(\eta)]^{\frac{t}{1+\zeta^{2}}} d \eta\right\}^{\frac{1+i \zeta}{t}},
$$

where $p \in \mathcal{P}$, and $\mathcal{P}$ is the class of analytic function with a positive real part, and $g \in \mathcal{S}^{*}$, where $\mathcal{S}^{*}$ is the well-known class of starlike function in the open unit disk $\mathbb{U}=\{z \in \mathbb{C}:|z|<1\}$. The numbers $t \geq 0$ and $\zeta$ are real and all powers are chosen so that the function remains single valued.

Other than the fact that $\mathcal{B}(t, \zeta, g)$ is univalent, we have little or no information on this family of functions. However, for some special cases, for example, if $\zeta=0$ and $g(z)=z$, we obtain the well-known class $\mathcal{B}(t)$ which satisfies the condition $\operatorname{Re} \frac{z^{1-t} f^{\prime}(z)}{(f(z))^{1-t}}>0, z \in \mathbb{U}$, where $f \in \mathcal{A}$, and $\mathcal{A}$ is the class of analytic functions in $\mathbb{U}$ having a Taylor series expansion of the form:

$$
f(z)=z+\sum_{k=2}^{\infty} a_{k} z^{k}, z \in \mathbb{U} .
$$

For $0 \leq \eta<1$, let $\mathcal{S}^{*}(\eta)$ and $\mathcal{C}(\eta)$ denote the classes of starlike functions of order $\eta$ and convex functions of order $\eta$, respectively. Babalola [2] introduced the class of functions $\mathcal{L}_{\lambda}(\eta)$, 
the so-called $\lambda$-pseudo-starlike functions of order $\eta$ as follows: a function $f \in \mathcal{A}$ is said to be in $\mathcal{L}_{\lambda}(\eta)$, with $0 \leq \eta<1, \lambda \geq 1$, if and only if it satisfies the inequality:

$$
\operatorname{Re} \frac{z\left(f^{\prime}(z)\right)^{\lambda}}{f(z)}>\eta, z \in \mathbb{U}
$$

If $\eta$ and $\vartheta$ are real numbers such that $0 \leq \eta<1<\vartheta$, let $\mathcal{S}(\eta, \vartheta)$ denote the class of functions $f \in \mathcal{A}$ satisfying the inequality:

$$
\eta<\operatorname{Re} \frac{z f^{\prime}(z)}{f(z)}<\vartheta, z \in \mathbb{U} .
$$

The class $\mathcal{S}(\eta, \vartheta)$ was introduced and first studied by Kuroki and Owa [3].

For $f \in \mathcal{A}$ given by (1) and $0<q<1$, the Jackson's $q$-derivative operator or $q$-difference operator for a function $f \in \mathcal{A}$ is defined by (see [4,5]):

$$
\mathfrak{D}_{q} f(z):= \begin{cases}f^{\prime}(0), & \text { if } z=0 \\ \frac{f(z)-f(q z)}{(1-q) z}, & \text { if } z \neq 0\end{cases}
$$

From the (3), if $f$ has the power series expansion (1), we can easily see that $\mathfrak{D}_{q} f(z)=1+\sum_{k=2}^{\infty}[k]_{q} a_{k} z^{k-1}$, for $z \neq 0$, where the $q$-integer number $[k]_{q}$ is defined by

$$
[k]_{q}:=\frac{1-q^{k}}{1-q}
$$

and note that $\lim _{q \rightarrow 1^{-}} \mathfrak{D}_{q} f(z)=f^{\prime}(z)$. Throughout this paper, we let denote:

$$
\left([k]_{q}\right)_{n}:=[k]_{q}[k+1]_{q}[k+2]_{q} \ldots[k+n-1]_{q} .
$$

For the function $f \in \mathcal{A}$ given by (1) and $h \in \mathcal{A}$ of the form $h(z)=z+\sum_{k=2}^{\infty} \Theta_{k} z^{k}$, the Hadamard product (or convolution) of these two functions is defined by

$$
H(z):=(f * h)(z):=z+\sum_{k=2}^{\infty} a_{k} \Theta_{k} z^{k}, z \in \mathbb{U} .
$$

Throughout our present discussion, to avoid repetition, we will assume that $-1 \leq B<A \leq 1$, and $\Theta_{k} \neq 0$ may be real or complex numbers.

For the functions $f$ and $g$ that are analytic in $\mathbb{U}$, we say that the function $f$ is subordinate to $g$ if there exists a function $w$, analytic in $\mathbb{U}$ with $w(0)=0$ and $|w(z)|<1, z \in \mathbb{U}$ such that $f=g \circ w$. We denote this subordination by $f \prec g$ or $f(z) \prec g(z)$. In particular, if the function $g$ is univalent in $\mathbb{U}$, the above subordination is equivalent to (see $[6,7]) f(0)=g(0)$ and $f(\mathbb{U}) \subset g(\mathbb{U})$.

Ma-Minda [8] considered that a given function $\psi$ is an analytic function such that:

(i) $\operatorname{Re} \psi>0, \mathbb{U}$;

(ii) $\psi(0)=1, \psi^{\prime}(0)>0$;

(iii) $\psi$ maps the open unit disc $\mathbb{U}$ onto a starlike region with respect to 1 and symmetric with respect to the real axis.

They also assumed that $\psi(z)=1+L_{1} z+L_{2} z^{2}+\ldots$, with $L_{1}>0$, and introduced and studied the following subclasses:

$$
\mathcal{S}^{*}(\psi):=\left\{f \in \mathcal{A}: \frac{z f^{\prime}(z)}{f(z)} \prec \psi(z)\right\}
$$


and:

$$
\mathcal{C}(\psi):=\left\{f \in \mathcal{A}: 1+\frac{z f^{\prime \prime}(z)}{f^{\prime}(z)} \prec \psi(z)\right\} .
$$

By choosing $\psi$ to map the unit disc onto some specific regions like parabolas, cardioid, lemniscate of Bernoulli, Booth lemniscate in the right-half of the complex plane, various interesting subclasses of starlike and convex functions can be obtained. For $-1 \leq B<A \leq 1$, we denote by $\mathcal{S}^{*}(A, B)$ and by $\mathcal{C}(A, B)$ the class of Janowski starlike functions and Janowski convex functions, defined by

$$
\mathcal{S}^{*}(A, B):=\left\{f \in \mathcal{A}: \frac{z f^{\prime}(z)}{f(z)} \prec \frac{1+A z}{1+B z},-1 \leq B<A \leq 1\right\},
$$

and:

$$
\mathcal{C}(A, B):=\left\{f \in \mathcal{A}: 1+\frac{z f^{\prime}(z)}{f(z)} \prec \frac{1+A z}{1+B z},-1 \leq B<A \leq 1\right\},
$$

respectively.

Motivated by the definition of $\lambda$-pseudo-starlike functions and unified subordination condition, we now introduce the following the class of functions:

Definition 1. For $-\frac{\pi}{2}<\alpha<\frac{\pi}{2}, \lambda \geq 1,0 \leq \beta \leq 1, t \geq 0, \gamma \in \mathbb{C} \backslash\{0\}$ and $H=f * h$ defined as in (4), we say that the function $f$ belongs to the class $\mathcal{P} \mathcal{S}_{\lambda}^{t}(\alpha, \beta ; \gamma ; \psi ; h ; A, B)$ if it satisfies the subordination condition:

$$
1+\frac{1+i \tan \alpha}{\gamma}\left[\frac{z^{1-t}\left[H^{\prime}(z)\right]^{\lambda}}{[(1-\beta) H(z)+\beta z]^{1-t}}-1\right] \prec \frac{(A+1) \psi(z)-(A-1)}{(B+1) \psi(z)-(B-1)},
$$

where " $\prec$ " denotes subordination, $\psi \in \mathcal{P}$, and $\psi(\mathbb{U})$ is the convex domain, while $\psi$ which has a power series expansion of the form:

$$
\psi(z)=1+L_{1} z+L_{2} z^{2}+L_{3} z^{3}+\ldots, z \in \mathbb{U}, L_{1} \neq 0,
$$

with $|\psi(z)|<\left|\frac{B-1}{B+1}\right|$ for all $z \in \mathbb{U}$.

Remark 1. Since $|\psi(z)|<\left|\frac{B-1}{B+1}\right|$ for all $z \in \mathbb{U}$, we note that the function $\mathrm{P}(z):=$ $\frac{(A+1) \psi(z)-(A-1)}{(B+1) \psi(z)-(B-1)}$ is analytic in $\mathbb{U}$ and can be expressed in power series in $\mathbb{U}$. In the above definition, the function $\psi$ is assumed to be convex since most of our main results require that the superordinate function P should be convex. However, we showed some applications where this condition could be relaxed.

Definition 2. For $-\frac{\pi}{2}<\alpha<\frac{\pi}{2}, \lambda \geq 1,0 \leq \beta \leq 1, t \geq 0, \gamma \in \mathbb{C} \backslash\{0\}$ and $H=f * h$ defined as in (4), we say that the function $f$ belongs to the class $\mathcal{S}_{\lambda}^{q}(t ; \alpha, \beta ; \gamma ; h, A, B)$ if it satisfies the subordination condition:

$$
1+\frac{1+i \tan \alpha}{\gamma}\left[\frac{z^{1-t}\left[\mathfrak{D}_{q} H(z)\right]^{\lambda}}{[(1-\beta) H(z)+\beta z]^{1-t}}-1\right] \prec \frac{(A+1) \hat{p}(z)-(A-1)}{(B+1) \hat{p}(z)-(B-1)},
$$

where $\hat{p}(z)=\frac{1+z}{1-q z}, q \in(0,1)$, satisfies the condition $|\hat{p}(z)|<\left|\frac{B-1}{B+1}\right|$ for all $z \in \mathbb{U}$. 
Remark 2. Since $\hat{p}(-1)=0, \hat{p}(1)=\frac{2}{1-q}>1$, and $\hat{p}(\bar{z})=\overline{\hat{p}(z)}, z \in \mathbb{U}$, it follows that the circular transform $\hat{p}$ maps the unit disc $\mathbb{U}$ onto the disc with diameter $\left[0 ; \frac{2}{1-q}\right]$ and symmetric with respect to the real axes. Therefore, the above assumption $|\hat{p}(z)|<\left|\frac{B-1}{B+1}\right|, z \in \mathbb{U}$, holds if and only if:

$$
\frac{2}{1-q} \leq\left|\frac{B-1}{B+1}\right|
$$

We also note that under this assumption, the function $\mathfrak{Q}(z):=\frac{(A+1) \hat{p}(z)-(A-1)}{(B+1) \hat{p}(z)-(B-1)}$ is analytic in $\mathbb{U}$ hence it can be expressed in power series in $\mathbb{U}$.

Remark 3. Several well-known classes can be seen as special cases of the class $\mathcal{P S}_{\lambda}^{t}(\alpha, \beta ; \gamma ; \psi ; h ; A, B)$ defined above (see also [9-11]). Now, we highlight only the recent works which are associated with a conic region:

(i) If $h(z)=z+\sum_{k=2}^{\infty} \frac{(2)_{k-1}}{(1)_{k-1}} z^{k}, \alpha=\beta=t=0, \gamma=1+0 i, \lambda=1$ and choosing $\psi(z)=$ $1+\frac{2}{\pi} \log \left(\frac{1+\sqrt{z}}{1-\sqrt{z}}\right)^{2}$, the class $\mathcal{P} \mathcal{S}_{\lambda}^{t}(\alpha, \beta ; \gamma ; \psi ; h ; A, B)$ reduces to the class UP $[A, B]$ introduced and studied by Malik et al. [12].

(ii) It can be easily seen that with the choice of $h(z)=z+\sum_{k=2}^{\infty} \frac{(b)_{k-1}}{(c)_{k-1}} z^{k}$, we get:

$$
\left[\mathcal{P} \mathcal{S}_{1}^{0}(0, \beta ; 1 ; \psi ; 1,-1)\right]_{\psi(z)=z+\sqrt{1+z^{2}}}=: \mathcal{M L}_{b}^{c}(\beta ; \psi)
$$

where $\mathcal{M L}_{b}^{c}(\beta ; \psi)$ is the class recently introduced and studied in [13]. Note that the function $\psi(z)=z+\sqrt{1+z^{2}}$ is starlike but not convex in $\mathbb{U}$.

Remark that, by the definition of the subordination, a function $H \in \mathcal{A}$ is said to be in $\mathcal{S}_{\lambda}^{q}(t ; \alpha, \beta ; \gamma ; h, A, B)$ if and only if there exists a function $w$ analytic in $\mathbb{U}$, with $w(0)=0$, and $|w(z)|<1$ for all $z \in \mathbb{U}$, such that:

$$
1+\frac{1+i \tan \alpha}{\gamma}\left[\frac{z^{1-t}\left[\mathfrak{D}_{q} H(z)\right]^{\lambda}}{[(1-\beta) H(z)+\beta z]^{1-t}}-1\right]=\frac{(A+1) w(z)+2+(A-1) q w(z)}{(B+1) w(z)+2+(B-1) q w(z)}
$$

where $q \in(0,1)$.

\section{Preliminaries}

In this section, we state the results that would be used to establish our main results which can be found in the standard text on univalent functions theory.

Lemma 1 ([14] (p. 56)). If the function $f \in \mathcal{A}$ given by (1) and g given by

$$
g(w)=w+\sum_{k=2}^{\infty} b_{k} w^{k}
$$

are inverse functions, then the coefficients $b_{k}$, for $k \geq 2$, are given by

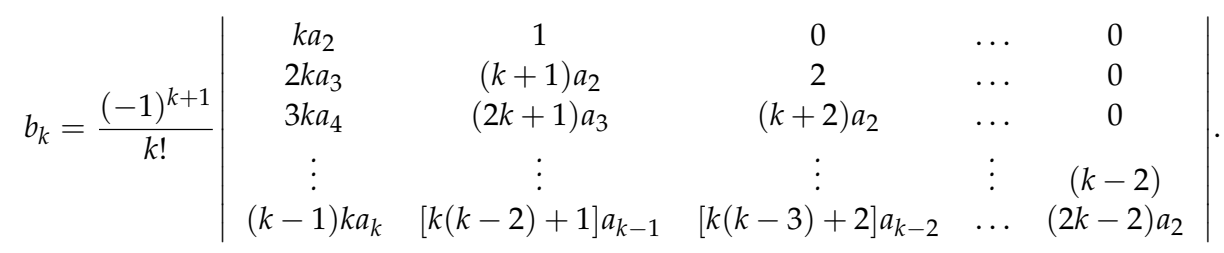


Remark 4. The elements of the above determinant (10) are given by

$$
\Lambda_{i j}= \begin{cases}{[(i-j+1) k+j-1] a_{i-j+2,},} & \text { if } i+1 \geq j, \\ 0, & \text { if } i+1<j .\end{cases}
$$

Lemma 2 ([7] (p. 41)). If $p(z)=1+\sum_{k=1}^{\infty} p_{k} z^{k} \in \mathcal{P}$, then $\left|p_{k}\right| \leq 2$ for all $k \geq 1$, and the inequality is sharp for $p_{\lambda}(z)=\frac{1+\lambda z}{1-\lambda z},|\lambda| \leq 1$.

Lemma 3 ([8]). If $p(z)=1+\sum_{k=1}^{\infty} p_{k} z^{k} \in \mathcal{P}$, and $v$ is a complex number, then:

$$
\left|p_{2}-v p_{1}^{2}\right| \leq 2 \max \{1 ;|2 v-1|\}
$$

and the result is sharp for the functions:

$$
p_{1}(z)=\frac{1+z}{1-z} \quad \text { and } \quad p_{2}(z)=\frac{1+z^{2}}{1-z^{2}} .
$$

Lemma 4 ([15] (Theorem VII)). Let $f(z)=\sum_{k=1}^{\infty} a_{k} z^{k}$ be analytic in $\mathbb{U}$ and $g(z)=\sum_{k=1}^{\infty} b_{k} z^{k}$ be analytic and convex in $\mathbb{U}$. If $f(z) \prec g(z)$, then $\left|a_{k}\right| \leq\left|b_{1}\right|$ for $k=1,2, \ldots$

Lemma 5 ([6] (Theorem 3.6.1)). Let the function $q$ be univalent in the open unit disc $\mathbb{U}$ and $\theta$ and $\phi$ be analytic in a domain $D$ containing $q(\mathbb{U})$ with $\phi(w) \neq 0$ when $w \in q(\mathbb{U})$. Set $Q(z)=z q^{\prime}(z) \phi(q(z)), k(z)=\theta(q(z))+Q(z)$. Suppose that:

1. $Q$ is starlike univalent in $\mathbb{U}$, and

2. $\operatorname{Re} \frac{z k^{\prime}(z)}{Q(z)}>0$, for $z \in \mathbb{U}$.

If:

$$
\theta(p(z))+z p^{\prime}(z) \phi(p(z)) \prec \theta(q(z))+z q^{\prime}(z) \phi(q(z)),
$$

then $p(z) \prec q(z)$ and $q$ is the best dominant.

\section{Conditions for Starlikeness Using Subordination}

In [6], the author presented new results in the theory of differential subordination with detailed proof. Motivated by the results presented in Chapter 4 of [6], we obtained the following result:

Theorem 1. Let the function $\psi \in \mathcal{A}$ be chosen such that the function:

$$
g(z):=\frac{(A+1) \psi(z)-(A-1)}{(B+1) \psi(z)-(B-1)}
$$

is convex univalent in $\mathbb{U}$, with:

$$
\operatorname{Re} g(z)>0, z \in \mathbb{U} \text {. }
$$

If the function $H=f * h \in \mathcal{A}$ satisfies the conditions:

$$
\begin{aligned}
& H^{\prime}(z) \neq 0, z \in \mathbb{U}, \\
& \frac{H(z)}{z} \neq-\frac{\beta}{1-\beta}, z \in \mathbb{U},
\end{aligned}
$$


then:

$$
\begin{gathered}
\frac{1+i \tan \alpha}{\gamma}\left[\frac{z^{1-t}\left[H^{\prime}(z)\right]^{\lambda}}{[(1-\beta) H(z)+\beta z]^{1-t}}-1\right] \times \\
\left\{2+\frac{1+i \tan \alpha}{\gamma}\left[\frac{z^{1-t}\left[H^{\prime}(z)\right]^{\lambda}}{[(1-\beta) H(z)+\beta z]^{1-t}}-1\right]\right. \\
+(t-1) \frac{(1-\beta) z H^{\prime}(z)+\beta z}{(1-\beta) H(z)+\beta z}+\frac{z^{1-t}\left[H^{\prime}(z)\right]^{\lambda}\left[(1-t)+\lambda\left[H^{\prime}(z)\right]^{-1} z H^{\prime \prime}(z)\right]}{z^{1-t}\left[H^{\prime}(z)\right]^{\lambda}-[(1-\beta) H(z)+\beta z]^{1-t}} \\
\left.-\frac{(1-t)[(1-\beta) H(z)+\beta z]^{-t}\left[(1-\beta) z H^{\prime}(z)+\beta z\right]}{z^{1-t}\left[H^{\prime}(z)\right]^{\lambda}-[(1-\beta) H(z)+\beta z]^{1-t}}\right\}+1 \prec k(z),
\end{gathered}
$$

where $k(z):=g^{2}(z)+z g^{\prime}(z)$, implies $f \in \mathcal{P} \mathcal{S}_{\lambda}^{t}(\alpha, \beta ; \gamma ; \psi ; h ; A, B)$. Moreover, the function $g$ is the best dominant of the left-hand side of (5).

Proof. If we define the function $p$ by

$$
p(z):=1+\frac{1+i \tan \alpha}{\gamma}\left[\frac{z^{1-t}\left[H^{\prime}(z)\right]^{\lambda}}{[(1-\beta) H(z)+\beta z]^{1-t}}-1\right], z \in \mathbb{U},
$$

then we form the assumptions (12) and (13), from which it follows that $p$ is analytic in $\mathbb{U}$. By a straight forward computation, we have:

$$
\begin{gathered}
z p^{\prime}(z)=[p(z)-1]\left[(t-1) \frac{(1-\beta) z H^{\prime}(z)+\beta z}{(1-\beta) H(z)+\beta z}\right. \\
+\frac{z^{1-t}\left[H^{\prime}(z)\right]^{\lambda}\left[(1-t)+\lambda\left[H^{\prime}(z)\right]^{-1} z H^{\prime \prime}(z)\right]}{z^{1-t}\left[H^{\prime}(z)\right]^{\lambda}-[(1-\beta) H(z)+\beta z]^{1-t}} \\
\left.-\frac{(1-t)[(1-\beta) H(z)+\beta z]^{-t}\left[(1-\beta) z H^{\prime}(z)+\beta z\right]}{z^{1-t}\left[H^{\prime}(z)\right]^{\lambda}-[(1-\beta) H(z)+\beta z]^{1-t}}\right],
\end{gathered}
$$

and thus, the subordination (14) is equivalent to:

$$
p^{2}(z)+z p^{\prime}(z) \prec k(z) .
$$

Setting:

$$
\theta(w):=w^{2} \text { and } \phi(w):=1,
$$

then $\theta$ and $\phi$ are analytic functions in $\mathbb{C}$, with $\phi(0) \neq 0$. Therefore:

$$
Q(z)=z g^{\prime}(z) \phi(g(z))=z g^{\prime}(z)
$$

and:

$$
k(z)=\theta(g(z))+Q(z)=g^{2}(z)+z g^{\prime}(z),
$$

and using the fact that $g$ is a convex univalent function in $\mathbb{U}$, it follows that:

$$
\begin{aligned}
& \operatorname{Re} \frac{z Q^{\prime}(z)}{Q(z)}=\operatorname{Re}\left(1+\frac{z g^{\prime \prime}(z)}{g^{\prime}(z)}\right)>0, z \in \mathbb{U}, \\
& Q^{\prime}(0)=g^{\prime}(0) \neq 0
\end{aligned}
$$

hence, $Q$ is a starlike univalent function in $\mathbb{U}$. Furthermore, the convexity of $g$ together with the assumption (11) implies:

$$
\operatorname{Re} \frac{z k^{\prime}(z)}{Q(z)}=\operatorname{Re}\left(2 g(z)+1+\frac{z g^{\prime \prime}(z)}{g^{\prime}(z)}\right)>0, z \in \mathbb{U},
$$


Since both of the conditions of Lemma 5 are satisfied, it follows that (15) implies $p(z) \prec g(z)$, and $g$ is the best dominant of $p$, which prove our conclusions.

Similar subordination conditions can be established for the class $\mathcal{S}_{\lambda}^{q}(t ; \alpha, \beta ; \gamma ; h, A, B)$, and here we choose to omit the details of the proof.

Theorem 2. Let the function $\hat{p}(z)=\frac{1+z}{1-q z}, q \in(0,1)$, be chosen such that the function:

$$
g(z):=\frac{(A+1) \hat{p}(z)-(A-1)}{(B+1) \hat{p}(z)-(B-1)}
$$

is convex univalent in $\mathbb{U}$, with:

$$
\operatorname{Re} g(z)>0, z \in \mathbb{U} \text {. }
$$

If the function $H=f * h \in \mathcal{A}$ satisfies the conditions:

$$
\begin{aligned}
& \mathfrak{D}_{q} H(z) \neq 0, z \in \mathbb{U}, \\
& \frac{H(z)}{z} \neq-\frac{\beta}{1-\beta}, z \in \mathbb{U},
\end{aligned}
$$

then:

$$
\begin{gathered}
\frac{1+i \tan \alpha}{\gamma}\left[\frac{z^{1-t}\left[\mathfrak{D}_{q} H(z)\right]^{\lambda}}{[(1-\beta) H(z)+\beta z]^{1-t}}-1\right]\left\{2+\frac{1+i \tan \alpha}{\gamma}\left[\frac{z^{1-t}\left[\mathfrak{D}_{q} H(z)\right]^{\lambda}}{[(1-\beta) H(z)+\beta z]^{1-t}}-1\right]\right. \\
+(t-1)\left(\frac{(1-\beta) z H^{\prime}(z)+\beta z}{(1-\beta) H(z)+\beta z}\right)+\frac{z^{1-t}\left[\mathfrak{D}_{q} H(z)\right]^{\lambda}\left[(1-t)+\lambda\left[\mathfrak{D}_{q} H(z)\right]^{-1} z\left(\mathfrak{D}_{q} H(z)\right)^{\prime}\right]}{z^{1-t}\left[\mathfrak{D}_{q} H(z)\right]^{\lambda}-[(1-\beta) H(z)+\beta z]^{1-t}} \\
\left.-\frac{(1-t)[(1-\beta) H(z)+\beta z]^{-t}\left(\left[(1-\beta) z H^{\prime}(z)+\beta z\right]\right)}{z^{1-t}\left[\mathfrak{D}_{q} H(z)\right]^{\lambda}-[(1-\beta) H(z)+\beta z]^{1-t}}\right\}+1 \prec k(z),
\end{gathered}
$$

where $k(z):=g^{2}(z)+z g^{\prime}(z)$, implies $f \in \mathcal{S}_{\lambda}^{q}(t ; \alpha, \beta ; \gamma ; h, A, B)$. Moreover, the function $g$ is the best dominant of the left-hand side of (7).

Theorem 3. If the function $H=f * h \in \mathcal{A}$ satisfies the conditions:

$$
\begin{aligned}
& H^{\prime}(z) \neq 0, z \in \mathbb{U}, \\
& \frac{H(z)}{z} \neq-\frac{\beta}{1-\beta}, z \in \mathbb{U},
\end{aligned}
$$

then:

$$
\begin{gathered}
\frac{z^{1-t}\left[H^{\prime}(z)\right]^{\lambda}}{[(1-\beta) H(z)+\beta z]^{1-t}}\left[\frac{\lambda z H^{\prime \prime}(z)}{H^{\prime}(z)}+(1-t)\left(1-\frac{(1-\beta) z H^{\prime}(z)+\beta z}{(1-\beta) H(z)+\beta z}\right)\right. \\
\left.+\frac{z^{1-t}\left[H^{\prime}(z)\right]^{\lambda}}{[(1-\beta) H(z)+\beta z]^{1-t}}\right] \prec k(z),
\end{gathered}
$$

where:

$$
k(z)=\frac{A^{2} z^{2}+(3 A-B) z+1}{(1+B z)^{2}},-1 \leq B<A \leq 1
$$

implies:

$$
\frac{z^{1-t}\left[H^{\prime}(z)\right]^{\lambda}}{[(1-\beta) H(z)+\beta z]^{1-t}} \prec \frac{1+A z}{1+B z},
$$

and this result is sharp. 
Proof. If we define the functions:

$$
g(z)=\frac{1+A z}{1+B z} \quad \text { and } \quad p(z)=\frac{z^{1-t}\left[H^{\prime}(z)\right]^{\lambda}}{[(1-\beta) H(z)+\beta z]^{1-t}},
$$

then $p$ is analytic in $\mathbb{U}$, and $g$ is a convex univalent function in $\mathbb{U}$ with $\operatorname{Re} g(z)>0$, $z \in \mathbb{U}$. Proceeding as in the proof of Theorem 1, we can establish the assertion of the Theorem 3.

If we let $\beta=t=0, \lambda=1$ and $h(z)=z+\sum_{k=2}^{\infty} z^{k}, z \in \mathbb{U}$, in the Theorem 3 , we obtain the next result:

Corollary 1 ([16] (Theorem 1$)$ ). If the function $f \in \mathcal{A}$ satisfies the condition $\frac{f(z)}{z} \neq 0, z \in \mathbb{U}$, then

$$
\frac{z^{2} f^{\prime \prime}(z)}{f(z)}+\frac{z f^{\prime}(z)}{f(z)} \prec k(z)
$$

where:

$$
k(z)=\frac{A^{2} z^{2}+(3 A-B) z+1}{(1+B z)^{2}},-1 \leq B<A \leq 1,
$$

implies $f \in \mathcal{S}^{*}(A, B)$.

For $A=1$ and $B=-1$, the function $k$ of Corollary 1 becomes $k(z)=\frac{z^{2}+4 z+1}{(1-z)^{2}}=$ $1+\frac{6 z}{(1-z)^{2}}$. If $z=e^{i \theta}, \theta \in[0,2 \pi]$, we get:

$$
k(z)=1-\frac{3}{2 \sin ^{2} \frac{\theta}{2}}, \theta \in(0,2 \pi) \backslash\{\pi\}
$$

thus, it follows that:

$$
k(\mathbb{U})=\hat{D}:=\mathbb{C} \backslash\left\{z \in \mathbb{C}: \operatorname{Re} z \leq-\frac{1}{2}, \operatorname{Im} z=0\right\} .
$$

According to the result of Corollary 1, in this present case, it follows that $f \in \mathcal{S}^{*}(1,-1)=\mathcal{S}^{*}$. On summarizing, we obtain the following sufficient condition for starlikeness obtained by Mocanu and Oros in [17]:

Example 1. If the function $f \in \mathcal{A}$ satisfies the condition $\frac{f(z)}{z} \neq 0, z \in \mathbb{U}$, and:

$$
\hat{D}:=\mathbb{C} \backslash\left\{z \in \mathbb{C}: \operatorname{Re} z \leq-\frac{1}{2}, \operatorname{Im} z=0\right\},
$$

then:

$$
\frac{z^{2} f^{\prime \prime}(z)}{f(z)}+\frac{z f^{\prime}(z)}{f(z)} \in \hat{D} \Rightarrow f \in \mathcal{S}^{*} .
$$


For $A=0$ and $B=-1$, the function $k$ of Corollary 1 becomes $k(z)=\frac{1+z}{(1-z)^{2}}$. If $z=e^{i \theta}, \theta \in[0,2 \pi]$, we get:

$$
\begin{gathered}
k(z)=\frac{1}{2} \frac{\cos \frac{\theta}{2}}{\sin ^{2} \frac{\theta}{2}}\left[\cos \left(\pi-\frac{\theta}{2}\right)+i \sin \left(\pi-\frac{\theta}{2}\right)\right]= \\
-\frac{1}{2 \tan ^{2} \frac{\theta}{2}}+\frac{i}{2 \tan \frac{\theta}{2}}, \theta \in[0,2 \pi] \backslash\{\pi\} .
\end{gathered}
$$

Denoting:

$$
\left\{\begin{array}{l}
u=-\frac{1}{2 t^{2}}, \\
v=\frac{1}{2 t}, t \in \mathbb{R},
\end{array}\right.
$$

and using the fact that $k(0)=1$ it follows that:

$$
k(\mathbb{U})=\left\{w=u+i v \in \mathbb{C}: u>-2 v^{2}\right\}=: \widetilde{D} .
$$

That is, $k(\mathbb{U})$ is the domain of $\mathbb{C}$ bounded by the parabola $u=-2 v^{2}$ that contains the point $z_{0}=1$.

Therefore, according to the result of Corollary 1, we have the following result:

Example 2 ([17]). If the function $f \in \mathcal{A}$ satisfies the condition $\frac{f(z)}{z} \neq 0, z \in \mathbb{U}$, and:

$$
\widetilde{D}:=\left\{w=u+i v \in \mathbb{C}: u>-2 v^{2}\right\}
$$

then:

$$
\frac{z^{2} f^{\prime \prime}(z)}{f(z)}+\frac{z f^{\prime}(z)}{f(z)} \in \widetilde{D} \Rightarrow f \in \mathcal{S}^{*}(1 / 2) .
$$

Corollary 2. If the function $H=f * h \in \mathcal{A}$ satisfies the conditions:

$$
\begin{aligned}
& H^{\prime}(z) \neq 0, z \in \mathbb{U}, \\
& \frac{H(z)}{z} \neq-\frac{\beta}{1-\beta}, z \in \mathbb{U},
\end{aligned}
$$

then:

$$
\begin{gathered}
\frac{z^{1-t}\left[H^{\prime}(z)\right]^{\lambda}}{[(1-\beta) H(z)+\beta z]^{1-t}}\left[\frac{\lambda z H^{\prime \prime}(z)}{H^{\prime}(z)}+(1-t)\left(1-\frac{(1-\beta) z H^{\prime}(z)+\beta z}{(1-\beta) H(z)+\beta z}\right)\right. \\
\left.+\frac{z^{1-t}\left[H^{\prime}(z)\right]^{\lambda}}{[(1-\beta) H(z)+\beta z]^{1-t}}\right] \prec 1+\delta z,
\end{gathered}
$$

where $\delta:=\delta(\mu):=\mu(3-\mu)$ and $0<\mu \leq 1$ implies:

$$
\frac{z^{1-t}\left[H^{\prime}(z)\right]^{\lambda}}{[(1-\beta) H(z)+\beta z]^{1-t}} \prec 1+\mu z .
$$

Proof. If we let $A=\mu$ and $B=0$, with $0<\mu \leq 1$, in Theorem 3 , then $k(z)=\mu^{2} z^{2}+3 \mu z+1$. For $|z|=1$, we get:

$$
|k(z)-1|=\mu|3+\mu z| \geq \mu(3-\mu)=: \delta .
$$

Since $k(0)=1$, from the above inequality, it follows that $1+\delta z \prec k(z)$ and the result follows from the Theorem 3. 
Letting $\beta=t=0, \lambda=1$ and $h(z)=z+\sum_{k=2}^{\infty} z^{k}$ in Corollary 2, we have the next special case:

Example 3. (see Corollary 4.3.2. in [6]) If the function $f \in \mathcal{A}$ satisfies the condition $\frac{f(z)}{z} \neq 0$, $z \in \mathbb{U}$, then:

$$
\frac{z^{2} f^{\prime \prime}(z)}{f(z)}+\frac{z f^{\prime}(z)}{f(z)} \prec 1+\delta z ，
$$

where $\delta:=\delta(\mu):=\mu(3-\mu)$ and $0<\mu \leq 1$, implies:

$$
\frac{z f^{\prime}(z)}{f(z)} \prec 1+\mu z \text {. }
$$

For $\mu=1$, the Example 3 reduces to the following result:

Example 4 ([6] (Example 4.3.1)). If $f \in \mathcal{A}$, then:

$$
\left|\frac{z f^{\prime}(z)}{f(z)}\left(1+\frac{f^{\prime \prime}(z)}{f^{\prime}(z)}\right)-1\right|<2, z \in \mathbb{U} \Rightarrow\left|\frac{z f^{\prime}(z)}{f(z)}-1\right|<1, z \in \mathbb{U} .
$$

4. Coefficients Estimates For the Functions of $\mathcal{P} \mathcal{S}_{\lambda}^{t}(\alpha, \beta ; \gamma ; \psi ; h ; A, B)$ and $\mathcal{S}_{\lambda}^{q}(t ; \alpha, \beta ; \gamma ; h, A, B)$

The class of all the functions of the class $\mathcal{P} \mathcal{S}_{\lambda}^{t}(\alpha, \beta ; \gamma ; \psi ; h ; A, B)$ are not univalent, so the inverse is not guaranteed. However, since $f^{\prime}(0)=1 \neq 0$ for all $f \in$ $\mathcal{P} \mathcal{S}_{\lambda}^{t}(\alpha, \beta ; \gamma ; \psi ; h ; A, B)$ and $f(0)=0$, there exists an inverse function in some small disk with a center at $w=0$ depending on the parameters involved.

Hereafter, unless otherwise mentioned $-\frac{\pi}{2}<\alpha<\frac{\pi}{2}, 0 \leq \lambda \leq 1, t \geq 0$ and $q \in(0,1)$. Additionally, let $g:=f^{-1}$ defined by $f^{-1}(f(z))=z, f\left(f^{-1}(z)\right)=w$ be the inverse of $f$ and:

$$
g(w)=f^{-1}(w)=w+\sum_{k=2}^{\infty} b_{k} w^{k},|w|<r_{0}, r_{0}>\frac{1}{4} .
$$

Furthermore, let $\Theta_{k}$ be the respective coefficients of $z^{k}$ in the power series expansion of $h$, as it appeared in the definition formula (4).

Theorem 4. If the function $f$ given by (1) and $g$ given by (9) are inverse functions and if $f \in \mathcal{P} \mathcal{S}_{\lambda}^{t}(\alpha, \beta ; \gamma ; \psi ; h ; A, B)$, then for the coefficients of $g=f^{-1}$, we have:

$$
\left|b_{2}\right| \leq \frac{|\gamma|(A-B)\left|L_{1}\right|}{2\left|\Theta_{2}\right|[2 \lambda+(t-1)(1-\beta)] \sec \alpha}
$$

and:

$$
\left|b_{3}\right| \leq \frac{\left|L_{1} \gamma\right|(A-B)}{2[3 \lambda+(t-1)(1-\beta)]\left|\Theta_{3}\right| \sec \alpha} \max \{1 ;|2 v-1|\}
$$

with:

$$
\begin{gathered}
v:=\frac{1}{4}\left[(B+1) L_{1}+2\left(1-\frac{L_{2}}{L_{1}}\right)+\frac{2 \gamma L_{1}(A-B)[3 \lambda+(t-1)(1-\beta)] \Theta_{3}}{\Theta_{2}^{2}(1+i \tan \alpha)[2 \lambda+(t-1)(1-\beta)]^{2}}\right. \\
\left.+\frac{\gamma L_{1}(A-B)\left\{4 \lambda[\lambda-1+(t-1)(1-\beta)]+(t-1)(t-2)(1-\beta)^{2}\right\}}{2(1+i \tan \alpha)[2 \lambda+(t-1)(1-\beta)]^{2}}\right] .
\end{gathered}
$$


Proof. If $f \in \mathcal{P} \mathcal{S}_{\lambda}^{t}(\alpha, \beta ; \gamma ; \psi ; h ; A, B)$, then by the definition of subordination, there exists a function $w$ analytic in $\mathbb{U}$, with $w(0)=0$ and $|w(z)|<1, z \in \mathbb{U}$, such that:

$$
1+\frac{1+i \tan \alpha}{\gamma}\left[\frac{z^{1-t}\left[H^{\prime}(z)\right]^{\lambda}}{[(1-\beta) H(z)+\beta z]^{1-t}}-1\right]=\frac{(A+1) \psi(w(z))-(A-1)}{(B+1) \psi(w(z))-(B-1)}, z \in \mathbb{U} .
$$

Thus, let $\ell \in \mathcal{P}$ be of the form: $\ell(z)=1+\sum_{k=1}^{\infty} p_{k} z^{k}$ and defined by

$$
\ell(z):=\frac{1+w(z)}{1-w(z)}, z \in \mathbb{U} .
$$

A simple computation shows that:

$$
\begin{gathered}
w(z)=\frac{\ell(z)-1}{\ell(z)+1}=\frac{p_{1} z+p_{2} z^{2}+p_{3} z^{3}+\ldots}{2+p_{1} z+p_{2} z^{2}+p_{3} z^{3}+\ldots} \\
=\frac{1}{2} p_{1} z+\frac{1}{2}\left(p_{2}-\frac{1}{2} p_{1}^{2}\right) z^{2}+\frac{1}{2}\left(p_{3}-p_{1} p_{2}+\frac{1}{4} p_{1}^{3}\right) z^{3}+\ldots, z \in \mathbb{U},
\end{gathered}
$$

and considering:

$$
\begin{gathered}
\frac{(A+1) \psi(w(z))-(A-1)}{(B+1) \psi(w(z))-(B-1)} \\
=1+\frac{L_{1} p_{1}(A-B)}{4} z+\frac{(A-B) L_{1}}{4}\left[p_{2}-p_{1}^{2}\left(\frac{(B+1) L_{1}+2\left(1-\frac{L_{2}}{L_{1}}\right)}{4}\right)\right] z^{2}+\ldots
\end{gathered}
$$

we have:

$$
\begin{array}{r}
\frac{z^{1-t}\left[H^{\prime}(z)\right]^{\lambda}}{[(1-\beta) H(z)+\beta z]^{1-t}}=1+\frac{\gamma}{1+i \tan \alpha}\left\{\frac{L_{1} p_{1}(A-B)}{4} z\right. \\
\left.+\frac{(A-B) L_{1}}{4}\left[p_{2}-p_{1}^{2}\left(\frac{(B+1) L_{1}+2\left(1-\frac{L_{2}}{L_{1}}\right)}{4}\right)\right] z^{2}+\ldots\right\}, z \in \mathbb{U} .
\end{array}
$$

The left hand side of (19) will be of the form:

$$
\begin{gathered}
\frac{z^{1-t}\left[H^{\prime}(z)\right]^{\lambda}}{[(1-\beta) H(z)+\beta z]^{1-t}}=1+[2 \lambda+(t-1)(1-\beta)] \Theta_{2} a_{2} z \\
+\left\{[3 \lambda+(t-1)(1-\beta)] \Theta_{3} a_{3}\right. \\
\left.+\left[2 \lambda[\lambda-1+(t-1)(1-\beta)]+\frac{(t-1)(t-2)(1-\beta)^{2}}{2}\right] \Theta_{2}^{2} a_{2}^{2}\right\} z^{2}+\ldots, z \in \mathbb{U},
\end{gathered}
$$

where $\Theta_{k}$ are the corresponding coefficients from the power series expansion of $h$, which may be real or complex.

From (19) and (20), we obtain:

$$
a_{2}=\frac{\gamma L_{1} p_{1}(A-B)}{4 \Theta_{2}(1+i \tan \alpha)[2 \lambda+(t-1)(1-\beta)]},
$$


and:

$$
\begin{gathered}
a_{3}=\frac{L_{1}(A-B) \gamma}{4(1+i \tan \alpha)[3 \lambda+(t-1)(1-\beta)] \Theta_{3}}\left[p_{2}-\frac{1}{4}\left((B+1) L_{1}+2\left(1-\frac{L_{2}}{L_{1}}\right)\right.\right. \\
\left.\left.+\frac{\gamma L_{1}(A-B)\left(4 \lambda[\lambda-1+(t-1)(1-\beta)]+(t-1)(t-2)(1-\beta)^{2}\right)}{2(1+i \tan \alpha)[2 \lambda+(t-1)(1-\beta)]^{2}}\right) p_{1}^{2}\right] .
\end{gathered}
$$

From (10), we see that $b_{2}=-a_{2}$, and applying Lemma 2 for (21), we obtain the inequality (16).

In addition, from (10), we have:

$$
\begin{aligned}
& b_{3}=\frac{(-1)^{4}}{3 !}\left|\begin{array}{cc}
3 a_{2} & 1 \\
6 a_{3} & 4 a_{2}
\end{array}\right|=2 a_{2}^{2}-a_{3}=\frac{\gamma^{2} L_{1}^{2} p_{1}^{2}(A-B)^{2}}{8 \Theta_{2}^{2}(1+i \tan \alpha)^{2}[2 \lambda+(t-1)(1-\beta)]^{2}} \\
& -\frac{L_{1}(A-B) \gamma}{4(1+i \tan \alpha)[3 \lambda+(t-1)(1-\beta)] \Theta_{3}}\left[p_{2}-\frac{1}{4}\left((B+1) L_{1}+2\left(1-\frac{L_{2}}{L_{1}}\right)\right.\right. \\
& \left.\left.+\frac{\gamma L_{1}(A-B)\left\{4 \lambda[\lambda-1+(t-1)(1-\beta)]+(t-1)(t-2)(1-\beta)^{2}\right\}}{2(1+i \tan \alpha)[2 \lambda+(t-1)(1-\beta)]^{2}}\right) p_{1}^{2}\right] \\
& =\frac{-L_{1}(A-B) \gamma}{4(1+i \tan \alpha)[3 \lambda+(t-1)(1-\beta)] \Theta_{3}}\left[p_{2}-\frac{1}{4}\left((B+1) L_{1}+2\left(1-\frac{L_{2}}{L_{1}}\right)\right.\right. \\
& +\frac{\gamma L_{1}(A-B)\left\{4 \lambda[\lambda-1+(t-1)(1-\beta)]+(t-1)(t-2)(1-\beta)^{2}\right\}}{2(1+i \tan \alpha)[2 \lambda+(t-1)(1-\beta)]^{2}}+ \\
& \left.\left.\frac{2 \gamma L_{1}(A-B)[3 \lambda+(t-1)(1-\beta)] \Theta_{3}}{\Theta_{2}^{2}(1+i \tan \alpha)[2 \lambda+(t-1)(1-\beta)]^{2}}\right) p_{1}^{2}\right],
\end{aligned}
$$

and using Lemma 2, we get (17), with $v$ given by (18).

Using a similar proof, we established the corresponding result for the class $\mathcal{S}_{\lambda}^{q}(t ; \alpha, \beta ; \gamma ; h, A, B)$ :

Theorem 5. If the function $f$ given by (1) and $g$ given by (9) are inverse functions and if $f \in$ $\mathcal{S}_{\lambda}^{q}(t ; \alpha, \beta ; \gamma ; h, A, B)$, then for the coefficients of $g=f^{-1}$ we have:

$$
\left|b_{2}\right| \leq \frac{|\gamma|(A-B)(q+1)}{2\left|\Theta_{2}\right|\left\{[2]_{q} \lambda+(t-1)(1-\beta)\right\} \sec \alpha},
$$

and:

$$
\left|b_{3}\right| \leq \frac{(q+1)|\gamma|(A-B)}{2\left\{[3]_{q} \lambda+(t-1)(1-\beta)\right\}\left|\Theta_{3}\right| \sec \alpha} \max \{1 ;|2 v-1|\},
$$

with:

$$
\begin{gathered}
v:=\frac{1}{4}\left[B(1+q)+(3-q)+\frac{2 \gamma(1+q)(A-B)\left\{[3]_{q} \lambda+(t-1)(1-\beta)\right\} \Theta_{3}}{\Theta_{2}^{2}(1+i \tan \alpha)\left([2]_{q} \lambda+(t-1)(1-\beta)\right)^{2}}\right. \\
\left.+\frac{\gamma(1+q)(A-B)\left\{(1+q) \lambda[(1+q)(\lambda-1)+2(t-1)(1-\beta)]+(t-1)(t-2)(1-\beta)^{2}\right\}}{2(1+i \tan \alpha)\left\{[2]_{q} \lambda+(t-1)(1-\beta)\right\}^{2}}\right] .
\end{gathered}
$$

Theorem 6. If the function $f$ given by (1) and $g$ given by (9) are inverse functions, and we let $H=f * h \in \mathcal{A}$ satisfy the inequality:

$$
\eta<\operatorname{Re} \frac{(B-1) \mathcal{L}(z)-(A-1)}{(B+1) \mathcal{L}(z)-(A+1)}<\vartheta, z \in \mathbb{U}, 0 \leq \eta<1<\vartheta,
$$


where:

$$
\mathcal{L}(z):=1+\frac{1+i \tan \alpha}{\gamma}\left[\frac{z^{1-t}\left[H^{\prime}(z)\right]^{\lambda}}{[(1-\beta) H(z)+\beta z]^{1-t}}-1\right] .
$$

Then for the coefficients of the inverse function $g=f^{-1}$, where $f$ satisfies the assumption (23), we have:

$$
\left|b_{2}\right| \leq \frac{|\gamma|(A-B)(\eta-\vartheta)}{\pi\left|\Theta_{2}\right|[2 \lambda+(t-1)(1-\beta)] \sec \alpha} \sin \frac{\pi(1-\eta)}{\eta-\vartheta},
$$

and:

$$
\begin{aligned}
& \left|b_{3}\right| \leq \frac{(A-B)(\eta-\vartheta) \sin \frac{\pi(1-\eta)}{\eta-\vartheta}}{\pi[3 \lambda+(t-1)(1-\beta)]\left|\Theta_{3}\right| \sec \alpha} \max \left\{1 ; \frac{\eta-\vartheta}{\pi} \sin \frac{\pi(1-\eta)}{\eta-\vartheta}\right. \\
& \mid B+1-\frac{2 \pi i}{\eta-\vartheta} \cot \frac{\pi(1-\eta)}{\eta-\vartheta}+\frac{2 \gamma(A-B)[3 \lambda+(t-1)(1-\beta)] \Theta_{3}}{\Theta_{2}^{2}(1+i \tan \alpha)[2 \lambda+(t-1)(1-\beta)]^{2}} \\
& \left.+\frac{\gamma(A-B)\left\{4 \lambda[\lambda-1+(t-1)(1-\beta)]+(t-1)(t-2)(1-\beta)^{2}\right\}}{2(1+i \tan \alpha)[2 \lambda+(t-1)(1-\beta)]^{2}} \mid\right\} .
\end{aligned}
$$

Proof. From the equivalent subordination condition proved by Kuroki and Owa in [3], we may rewrite the conditions (23) in the following form:

$$
\frac{(B-1) \mathcal{L}(z)-(A-1)}{(B+1) \mathcal{L}(z)-(A+1)} \prec 1+\frac{\eta-\vartheta}{\pi} i \log \left(\frac{1-e^{2 \pi i((1-\eta) /(\eta-\vartheta))} z}{1-z}\right)=: \mathcal{T}(z),
$$

that is equivalent to:

$$
\mathcal{L}(z) \prec \frac{(A+1) \mathcal{T}(z)-(A-1)}{(B+1) \mathcal{T}(z)-(B-1)},
$$

i.e., $f \in \mathcal{P} \mathcal{S}_{\lambda}^{t}(\alpha, \beta ; \gamma ; \mathcal{T} ; h ; A, B)$. Furthermore, we note that the function $\mathcal{T}$ given by

$$
\mathcal{T}(z)=1+\frac{\eta-\vartheta}{\pi} i \log \left(\frac{1-e^{2 \pi i((1-\eta) /(\eta-\vartheta))} z}{1-z}\right)
$$

maps the open unit disk $\mathbb{U}$ onto a convex domain and is of the form:

$$
\mathcal{T}(z)=1+\sum_{k=1}^{\infty} L_{k} z^{k}, z \in \mathbb{U}
$$

where $L_{k}=\frac{\eta-\vartheta}{k \pi} i\left(1-e^{2 k \pi i((1-\eta) /(\eta-\vartheta))}\right), k \geq 1$. Substituting the values of $L_{1}$ and $L_{2}$ in Theorem 4 , we obtain the assertion of our theorem.

If we let $\alpha=\beta=t=0, \lambda=\gamma=A=1, B=-1$ and $h(z)=z+\sum_{k=2}^{\infty} z^{k}$ in Theorem 6, we obtain the result obtained by Sim and Kwon [18]:

Corollary 3 ([18] (Corollary 1)). If the function $f$ given by (1) and $g$ given by (9) are inverse functions and if $f \in \mathcal{S}(\eta, \vartheta)$, with $0 \leq \eta<1<\vartheta$, is defined by (2), then:

$$
\left|b_{2}\right| \leq \frac{2(\eta-\vartheta)}{\pi} \sin \frac{\pi(1-\eta)}{\eta-\vartheta}
$$

and:

$$
\left|b_{3}\right| \leq \frac{\eta-\vartheta}{\pi} \sin \frac{\pi(1-\eta)}{\eta-\vartheta} \max \left\{1 ;\left|\frac{1}{2}-3 \frac{\eta-\vartheta}{\pi} i+\left(\frac{1}{2}+3 \frac{\eta-\vartheta}{\pi} i\right) e^{2 \pi i \frac{1-\eta}{\eta-\vartheta}}\right|\right\} .
$$


The impact of the well-known Janowski function:

$$
\mathcal{J}(z):=1+\frac{2}{\pi^{2}}\left(\log \frac{1+\sqrt{z}}{1-\sqrt{z}}\right)^{2}, z \in \mathbb{U},
$$

was recently studied by Malik et al. [12]. Following the same steps as in Theorem 1 of [19], we obtain:

$$
\mathcal{J}(z)=1+\frac{8}{\pi^{2}} z+\frac{16}{3 \pi^{2}} z^{2}+\ldots, z \in \mathbb{U} .
$$

Replacing the values of $L_{1}, L_{2}$ and $L_{3}$ of Theorem 4 with the corresponding coefficients of the power series (26), we obtain the next result:

Theorem 7. If the function $f$ given by (1) and $g$ given by (9) are inverse functions and if $f \in \mathcal{P S}_{\lambda}^{t}(\alpha, \beta ; \gamma ; \mathcal{J} ; h ; A, B)$, with $\mathcal{J}$ defined as in (25), then for the coefficients of $g=f^{-1}$, we have:

$$
\left|b_{2}\right| \leq \frac{4|\gamma|(A-B)}{\pi^{2}\left|\Theta_{2}\right|[2 \lambda+(t-1)(1-\beta)] \sec \alpha},
$$

and:

$$
\begin{gathered}
\left|b_{3}\right| \leq \frac{4|\gamma|(A-B)}{\pi^{2}[3 \lambda+(t-1)(1-\beta)]\left|\Theta_{3}\right| \sec \alpha} \max \left\{1 ; \frac{4}{\pi^{2}} \mid\left(B+1-\frac{\pi^{2}}{6}\right)\right. \\
+\frac{\gamma(A-B)\left\{4 \lambda[\lambda-1+(t-1)(1-\beta)]+(t-1)(t-2)(1-\beta)^{2}\right\}}{2(1+i \tan \alpha)[2 \lambda+(t-1)(1-\beta)]^{2}} \\
\left.+\frac{2 \gamma(A-B)[3 \lambda+(t-1)(1-\beta)] \Theta_{3}}{\Theta_{2}^{2}(1+i \tan \alpha)[2 \lambda+(t-1)(1-\beta)]^{2}} \mid\right\} .
\end{gathered}
$$

If we let $\alpha=\beta=t=0, \lambda=\gamma=1$ and $h(z)=z+\sum_{k=2}^{\infty} \frac{(2)_{k-1}}{(1)_{k-1}} z^{k}, z \in \mathbb{U}$, in Theorem 7, we obtain the following result:

Corollary 4 ([12] (Theorem 4)). If the function $f$ given by (1) and g given by (9) are inverse functions and if $f \in \mathcal{P} \mathcal{S}_{1}^{0}(0,0 ; 1 ; \mathcal{J} ; A, B)$, with $\mathcal{J}$ defined as in (25), then for the coefficients of $g=f^{-1}$, we have:

$$
\left|b_{k}\right| \leq \frac{4(A-B)}{k(k-1) \pi^{2}}, k=2,3
$$

We need the following result to establish the coefficient estimate $\left|a_{k}\right|$ of the classes $\mathcal{P} \mathcal{S}_{1}^{0}(\alpha, \beta ; \gamma ; \psi ; h ; A, B)$ and $\mathcal{S}_{1}^{q}(0 ; \alpha, \beta ; \gamma ; A, B)$.

Lemma 6. Let the function $\mathrm{P}(z):=\frac{(A+1) \psi(z)-(A-1)}{(B+1) \psi(z)-(B-1)}$ be convex in $\mathbb{U}$ where the function $\psi$ is defined as in (6). If $p(z)=1+\sum_{k=1}^{\infty} p_{k} z^{k}$ is analytic in $\mathbb{U}$ and satisfies the subordination condition:

$$
p(z) \prec \frac{(A+1) \psi(z)-(A-1)}{(B+1) \psi(z)-(B-1)},
$$

then:

$$
\left|p_{k}\right| \leq \frac{\left|L_{1}\right|(A-B)}{2}, k \geq 1 .
$$

Proof. If the function $\psi$ has the power series expansion (6), then:

$$
\mathrm{P}(z)=1+\mathrm{P}^{\prime}(0) z+\cdots=1+\frac{(A-B) L_{1}}{2} z+\ldots, z \in \mathbb{U} .
$$


Since the subordination relation is invariant regarding a translation, the assumption (27) is equivalent to:

$$
p(z)-1 \prec \mathrm{P}(z)-1 \text {. }
$$

In addition, because the convexity of $\mathrm{P}$ implies the convexity of $\mathrm{P}-1$, from Lemma 4 , it follows the conclusion (28):

Theorem 8. Let $f \in \mathcal{P} \mathcal{S}_{1}^{0}(\alpha, \beta ; \gamma ; \psi ; h ; A, B)$. Then:

$$
\left|a_{2}\right| \leq \frac{|\gamma|(A-B)\left|L_{1}\right|}{2(\beta+1)\left|\Theta_{2}\right| \sec \alpha}=: s_{2}
$$

and:

$$
\left|a_{k}\right|\left|\Theta_{k}\right| \leq \frac{|\gamma|(A-B)\left|L_{1}\right|}{2(\beta-1+k) \sec \alpha}\left[1+(1-\beta) \sum_{j=2}^{k-1} s_{j}\right]=: s_{k}
$$

for $k \geq 3$

Proof. By the definition of $\mathcal{P} \mathcal{S}_{1}^{0}(\alpha, \beta ; \gamma ; \psi ; h ; A, B)$, we have:

$$
1+\frac{1+i \tan \alpha}{\gamma}\left[\frac{z H^{\prime}(z)}{(1-\beta) H(z)+\beta z}-1\right]=: p(z),
$$

where $p(z)=1+\sum_{k=1}^{\infty} p_{k} z^{k}$ is analytic in $\mathbb{U}$ and satisfies the subordination condition: $p(z) \prec \frac{(A+1) \psi(z)-(A-1)}{(B+1) \psi(z)-(B-1)}$. The relation (29) can be rewritten as

$$
(1+i \tan \alpha) \sum_{k=2}^{\infty}[(\beta-1)+k] \Theta_{k} a_{k} z^{k}=\gamma\left[z+\sum_{k=2}^{\infty}(1-\beta) \Theta_{k} a_{k} z^{k}\right]\left(\sum_{k=1}^{\infty} p_{k} z^{k}\right), z \in \mathbb{U},
$$

and equating the coefficient of $z^{k}$, we get:

$$
\begin{gathered}
(1+i \tan \alpha)[(\beta-1)+k] \Theta_{k} a_{k} \\
=\gamma\left[p_{k-1}+p_{k-2}(1-\beta) \Theta_{2} a_{2}+\cdots+p_{1}(1-\beta) \Theta_{k-1} a_{k-1}\right]
\end{gathered}
$$

for $k \geq 3$, and for $k=2$ we have:

$$
(1+i \tan \alpha)(\beta+1) \Theta_{2} a_{2}=\gamma p_{1} .
$$

It follows that:

$$
\begin{aligned}
\left|a_{k}\right|\left|\Theta_{k}\right| & \leq \frac{|\gamma|}{[(\beta-1)+k] \sec \alpha}\left[\left|p_{k-1}\right|+\sum_{j=1}^{k-2}(1-\beta)\left|\Theta_{k-j}\right|\left|a_{k-j}\right|\left|p_{j}\right|\right], k \geq 3, \\
\left|a_{2}\right|\left|\Theta_{2}\right| & =\frac{|\gamma|\left|p_{1}\right|}{(\beta+1) \sec \alpha},
\end{aligned}
$$

and using (28) in the above inequality, it follows that:

$$
\begin{aligned}
\left|a_{k}\right|\left|\Theta_{k}\right| & \leq \frac{\left|\gamma(A-B) L_{1}\right|}{2[(\beta-1)+k] \sec \alpha}\left[1+(1-\beta) \sum_{j=1}^{k-2}\left|\Theta_{k-j}\right|\left|a_{k-j}\right|\right] \\
& =\frac{\left|\gamma(A-B) L_{1}\right|}{2[(\beta-1)+k] \sec \alpha}\left[1+(1-\beta) \sum_{j=2}^{k-1}\left|\Theta_{j}\right|\left|a_{j}\right|\right]=: s_{k}, k \geq 3, \\
\left|a_{2}\right|\left|\Theta_{2}\right| & \leq \frac{|\gamma|(A-B)\left|L_{1}\right|}{2(\beta+1) \sec \alpha}=: s_{2},
\end{aligned}
$$


which proves our conclusions.

For the proof of the next result, we will use the well-known Parseval's identity (see also [20] (Theorem 15, p. 505)): if $F$ and $G$ are two square-integrable complex functions on the interval $[-\pi, \pi]$, with Fourier series $F(x)=\sum_{n=-\infty}^{\infty} a_{n} e^{i n x}$ and $G(x)=\sum_{n=-\infty}^{\infty} b_{n} e^{i n x}$, then:

$$
\sum_{n=-\infty}^{\infty} a_{n} \bar{b}_{n}=\frac{1}{2 \pi} \int_{-\pi}^{\pi} F(x) \bar{G}(x) d x
$$

Theorem 9. Let $f \in \mathcal{S}_{1}^{q}(0 ; \alpha, \beta ; \gamma ; A, B)$, that is the class $\mathcal{S}_{\lambda}^{q}(t ; \alpha, \beta ; \gamma ; h, A, B)$ given by Definition 2 for $\lambda=1$ and $t=0$, then:

$$
\left|a_{2}\right| \leq \frac{|\gamma|(A-B)(1+q)}{2\left(\beta-1+[2]_{q}\right)\left|\Theta_{2}\right| \sec \alpha}
$$

and for $j \geq 3$ we have:

$$
\begin{gathered}
\left|a_{j}\right| \leq \frac{|\gamma|(A-B)(1+q)}{2(\beta+1) \sec \alpha\left|\Theta_{j}\right|} \\
\cdot \prod_{k=1}^{j-2} \frac{\left|(A-B)(1+q) \gamma(1-\beta)-(1+i \tan \alpha)\left(\beta+[k-1]_{q}+1\right)[B(1+q)+(1-q)]\right|}{2\left(\beta+[k]_{q}+1\right) \sec \alpha} .
\end{gathered}
$$

Proof. From (8), we have:

$$
\begin{gathered}
2(1+i \tan \alpha)\left\{z \mathfrak{D}_{q} H(z)-[(1-\beta) H(z)+\beta z]\right\}= \\
\{(A-B) \gamma(1+q)[(1-\beta) H(z)+\beta z] \\
\left.-[B(1+q)+(1-q)](1+i \tan \alpha)\left[z \mathfrak{D}_{q} H(z)-[(1-\beta) H(z)+\beta z]\right]\right\} w(z),
\end{gathered}
$$

hence:

$$
\begin{gathered}
\sum_{k=2}^{\infty} 2(1+i \tan \alpha)\left([k]_{q}+\beta-1\right) a_{k} \Theta_{k} z^{k} \\
=\left\{(A-B) \gamma(1+q) z+\sum_{k=2}^{\infty}[(1-\beta)(A-B)(1+q) \gamma\right. \\
\left.\left.-[B(1+q)+(1-q)](1+i \tan \alpha)\left([k]_{q}+\beta-1\right)\right] \Theta_{k} a_{k} z^{k}\right\} w(z) .
\end{gathered}
$$

We will use the technique employed by Clunie which consists of breaking the summation on the right side into two parts which are $\sum_{k=2}^{j-1}$ and $\sum_{k=j}^{\infty}$, and to observe that the 
multiplication by $w(z)=\sum_{k=2}^{\infty} \omega_{k} z^{k},|w(z)|<1,|z|<1$, of the second sum gives terms with exponents that exceeds $j$.

$$
\begin{gathered}
\sum_{k=2}^{j} 2(1+i \tan \alpha)\left([k]_{q}+\beta-1\right) a_{k} \Theta_{k} z^{k}+\sum_{k=j+1}^{\infty} 2(1+i \tan \alpha)\left([k]_{q}+\beta-1\right) a_{k} \Theta_{k} z^{k} \\
=\left\{(A-B) \gamma(1+q) z+\sum_{k=2}^{j-1}[(1-\beta)(A-B)(1+q) \gamma\right. \\
\left.\left.-[B(1+q)+(1-q)](1+i \tan \alpha)\left([k]_{q}+\beta-1\right)\right] \Theta_{k} a_{k} z^{k}\right\} w(z) \\
+\sum_{k=j}^{\infty}[(1-\beta)(A-B)(1+q) \gamma \\
\left.-[B(1+q)+(1-q)](1+i \tan \alpha)\left([k]_{q}+\beta-1\right)\right] \Theta_{k} a_{k} z^{k} w(z) .
\end{gathered}
$$

Rearranging the last summation, we can write:

$$
\begin{gathered}
\sum_{k=2}^{j} 2(1+i \tan \alpha)\left([k]_{q}+\beta-1\right) a_{k} \Theta_{k} z^{k}+\sum_{k=j+1}^{\infty} d_{k} z^{k} \\
=\left\{(A-B) \gamma(1+q) z+\sum_{k=2}^{j-1}[(1-\beta)(A-B)(1+q) \gamma\right. \\
\left.\left.-[B(1+q)+(1-q)](1+i \tan \alpha)\left([k]_{q}+\beta-1\right)\right] \Theta_{k} a_{k} z^{k}\right\} w(z),
\end{gathered}
$$

therefore:

$$
\begin{gathered}
\left|\sum_{k=2}^{j} 2(1+i \tan \alpha)\left([k]_{q}+\beta-1\right) a_{k} \Theta_{k} z^{k}+\sum_{k=j+1}^{\infty} d_{k} z^{k}\right|= \\
\mid \gamma(A-B)(1+q) z+\sum_{k=2}^{j-1}\{(1-\beta)(A-B)(1+q) \gamma \\
\left.-[B(1+q)+(1-q)](1+i \tan \alpha)\left([k]_{q}+\beta-1\right)\right\} \Theta_{k} a_{k} z^{k}|| w(z) \mid .
\end{gathered}
$$

The second series on the left hand side of the above equation is given by

$$
\begin{aligned}
\sum_{k=j+1}^{\infty} d_{k} z^{k}= & \sum_{k=j+1}^{\infty} 2(1+i \tan \alpha)\left([k]_{q}+\beta-1\right) a_{k} \Theta_{k} z^{k}-\left(\sum_{k=j}^{\infty}\{(1-\beta)(A-B)(1+q) \gamma\right. \\
& \left.\left.-[B(1+q)+(1-q)](1+i \tan \alpha)\left([k]_{q}+\beta-1\right)\right\} \Theta_{k} a_{k} z^{k}\right) w(z)
\end{aligned}
$$

which is obviously convergent in $\mathbb{U}$. If we let $z=r e^{i \theta}, r<1$, since $|w(z)|<1, z \in \mathbb{U}$, and applying Parseval's formula on the both sides of the above relation, then by letting $r \rightarrow 1^{-}$ we obtain:

$$
\begin{gathered}
4 \sec ^{2} \alpha\left([j]_{q}+\beta-1\right)^{2}\left|\Theta_{j}\right|^{2}\left|a_{j}\right|^{2} \leq(A-B)^{2}(1+q)^{2}|\gamma|^{2} \\
+\sum_{k=2}^{j-1}\left\{\left|(1-\beta)(A-B)(1+q) \gamma+(1+i \tan \alpha) \cdot[B(1+q)+(1-q)]\left([k]_{q}+\beta-1\right)\right|^{2}\right. \\
\left.-4 \sec ^{2} \alpha\left([k]_{q}+\beta-1\right)^{2}\right\}\left|\Theta_{k}\right|^{2}\left|a_{k}\right|^{2}, j \geq 2 .
\end{gathered}
$$


For $j=2$, from (31) it follows that:

$$
\left|a_{2}\right| \leq \frac{|\gamma|(A-B)(1+q)}{2 \sec \alpha\left(\beta-1+[2]_{q}\right)\left|\Theta_{2}\right|} .
$$

For $j=3$, from (31) we obtain:

$$
\begin{gathered}
4 \sec ^{2} \alpha\left([3]_{q}+\beta-1\right)^{2}\left|\Theta_{3}\right|^{2}\left|a_{3}\right|^{2} \leq(A-B)^{2}(1+q)^{2}|\gamma|^{2} \\
+\left\{\left|(1-\beta)(A-B)(1+q) \gamma+(1+i \tan \alpha) \cdot[B(1+q)+(1-q)]\left([2]_{q}+\beta-1\right)\right|^{2}\right. \\
\left.-4 \sec ^{2} \alpha\left([2]_{q}+\beta-1\right)^{2}\right\}\left|\Theta_{2}\right|^{2}\left|a_{2}\right|^{2}, j \geq 2 .
\end{gathered}
$$

Now, substituting in the right hand side of the above relation, the value of $\left|a_{2}\right|^{2}$ with its majorant given by (32), we have:

$$
\begin{gathered}
4 \sec ^{2} \alpha\left([3]_{q}+\beta-1\right)^{2}\left|\Theta_{3}\right|^{2}\left|a_{3}\right|^{2} \leq\left[(A-B)^{2}(1+q)^{2}|\gamma|^{2}\right. \\
+\left\{\left|(1-\beta)(A-B)(1+q) \gamma+(1+i \tan \alpha)[B(1+q)+(1-q)]\left([2]_{q}+\beta-1\right)\right|^{2}\right. \\
\left.\left.-4 \sec ^{2} \alpha\left([2]_{q}+\beta-1\right)^{2}\right\}\left|\Theta_{2}\right|^{2} \frac{|\gamma|^{2}(A-B)^{2}(1+q)^{2}}{4 \sec ^{2} \alpha\left(\beta-1+[2]_{q}\right)^{2}\left|\Theta_{2}\right|^{2}}\right],
\end{gathered}
$$

that implies:

$$
\begin{gathered}
\left|a_{3}\right|^{2} \leq \frac{(A-B)^{2}(1+q)^{2}|\gamma|^{2}}{4 \sec ^{2} \alpha\left([3]_{q}+\beta-1\right)^{2}\left|\Theta_{3}\right|^{2}}[1+ \\
\left.\frac{\left|(1-\beta)(A-B)(1+q) \gamma+(1+i \tan \alpha) \cdot[B(1+q)+(1-q)]\left([2]_{q}+\beta-1\right)\right|^{2}}{4 \sec ^{2} \alpha\left(\beta-1+[2]_{q}\right)^{2}}-1\right] .
\end{gathered}
$$

Therefore, (30) is true for $j=3$. Following the same steps as in [21], we can prove the assertion (30) of our theorem:

If we take $\lambda=1=\gamma, t=\beta=0, h(z)=z+\sum_{k=2}^{\infty} z^{k}, z \in \mathbb{U}$, and $q \rightarrow 1^{-}$in Theorem 9, we obtain the following result:

Corollary 5 ([22] (Theorem 1$)$ ). If $f \in \mathcal{A}$ satisfies the subordination:

$$
(1+i \tan \alpha) \frac{z f^{\prime}(z)}{f(z)}-i \tan \alpha \prec \frac{1+A z}{1+B z},
$$

then:

$$
\left|a_{j}\right| \leq \prod_{k=0}^{j-2} \frac{\left|(A-B) e^{-i \alpha} \cos \alpha-k B\right|}{(k+1)}, j \geq 2
$$

where $|\alpha|<\pi / 2$.

For $A=1-2 \eta, 0 \leq \eta<1, B=-1$ and $q \rightarrow 1^{-}$Corollary 5 reduces to the next special case:

Example 5 ([23] (Theorem 1)). If $f \in \mathcal{A}$ satisfy the inequality:

$$
\operatorname{Re}\left\{e^{i \alpha} \frac{z f^{\prime}(z)}{f(z)}\right\}>\eta \cos \alpha, z \in \mathbb{U},
$$


with $|\alpha|<\pi / 2$, then:

$$
\left|a_{j}\right| \leq \prod_{k=0}^{j-2} \frac{\left|2(1-\eta) e^{-i \alpha} \cos \alpha+k\right|}{k+1}, j \geq 2
$$

The coefficient estimates of (33) are sharp.

Remark 5. Several well-known results can be obtained as the special case of Theorems 8 and 9 , and we refer to the papers $[21,24]$ in addition to the references provided therein.

5. Solution to Fekete-Szegó Problem for the Functions of $\mathcal{P} \mathcal{S}_{\lambda}^{t}(\alpha, \beta ; \gamma ; \psi ; h ; A, B)$ and $\mathcal{S}_{\lambda}^{q}(t ; \alpha, \beta ; \gamma ; h, A, B)$

We will give the solution of the Fekete-Szegó problem for the functions that belong to the classes we defined in the first section.

Theorem 10. If $f(z)=z+a_{2} z^{2}+a_{3} z^{3}+\cdots \in \mathcal{P} \mathcal{S}_{\lambda}^{t}(\alpha, \beta ; \gamma ; \psi ; h ; A, B)$, then for all $\mu \in \mathbb{C}$, we have:

$$
\left|a_{3}-\mu a_{2}^{2}\right| \leq \frac{\left|L_{1}\right|(A-B)|\gamma|}{2[3 \lambda+(t-1)(1-\beta)]\left|\Theta_{3}\right| \sec \alpha} \max \{1 ;|2 \rho-1|\},
$$

where $\rho$ is given by

$$
\begin{gathered}
\rho:=\frac{1}{4}\left[(B+1) L_{1}+2\left(1-\frac{L_{2}}{L_{1}}\right)+\frac{\mu \gamma L_{1}(A-B)[3 \lambda+(t-1)(1-\beta)] \Theta_{3}}{\Theta_{2}^{2}(1+i \tan \alpha)[2 \lambda+(t-1)(1-\beta)]^{2}}\right. \\
\left.+\frac{\gamma L_{1}(A-B)\left\{4 \lambda[\lambda-1+(t-1)(1-\beta)]+(t-1)(t-2)(1-\beta)^{2}\right\}}{2(1+i \tan \alpha)[2 \lambda+(t-1)(1-\beta)]^{2}}\right] .
\end{gathered}
$$

The inequality is sharp for each $\mu \in \mathbb{C}$.

Proof. If $f \in \mathcal{P} \mathcal{S}_{\lambda}^{t}(\alpha, \beta ; \gamma ; \psi ; h ; A, B)$, in view of the relations (21) and (22), for $\mu \in \mathbb{C}$, we have:

$$
\begin{gathered}
\left|a_{3}-\mu a_{2}^{2}\right|=\mid \frac{L_{1}(A-B) \gamma}{4(1+i \tan \alpha)[3 \lambda+(t-1)(1-\beta)] \Theta_{3}}\left[p_{2}-\frac{1}{4}\left((B+1) L_{1}+2\left(1-\frac{L_{2}}{L_{1}}\right)\right.\right. \\
\left.\left.+\frac{\gamma L_{1}(A-B)\left\{4 \lambda[\lambda-1+(t-1)(1-\beta)]+(t-1)(t-2)(1-\beta)^{2}\right\}}{2(1+i \tan \alpha)[2 \lambda+(t-1)(1-\beta)]^{2}}\right) p_{1}^{2}\right] \\
-\frac{\mu \gamma^{2} L_{1}^{2} p_{1}^{2}(A-B)^{2}}{16 \Theta_{2}^{2}(1+i \tan \alpha)^{2}[2 \lambda+(t-1)(1-\beta)]^{2}} \mid \\
=\mid \frac{L_{1}(A-B) \gamma}{4(1+i \tan \alpha)[3 \lambda+(t-1)(1-\beta)] \Theta_{3}}\left[p_{2}-\frac{p_{1}^{2}}{2}+\frac{1}{4} p_{1}^{2}\left(\frac{2 L_{2}}{L_{1}}-(B+1) L_{1}\right.\right. \\
-\frac{\gamma L_{1}(A-B)\left\{4 \lambda[\lambda-1+(t-1)(1-\beta)]+(t-1)(t-2)(1-\beta)^{2}\right\}}{2(1+i \tan \alpha)[2 \lambda+(t-1)(1-\beta)]^{2}} \\
\left.\left.-\frac{\mu \gamma L_{1}(A-B)[3 \lambda+(t-1)(1-\beta)] \Theta_{3}}{\Theta_{2}^{2}(1+i \tan \alpha)[2 \lambda+(t-1)(1-\beta)]^{2}}\right)\right] \mid \\
\leq \frac{\left|L_{1}\right|(A-B)|\gamma|}{4 \sec \alpha[3 \lambda+(t-1)(1-\beta)]\left|\Theta_{3}\right|}\left[2+\frac{1}{4}\left|p_{1}\right|^{2}\left(\mid \frac{2 L_{2}}{L_{1}}-(B+1) L_{1}\right.\right. \\
-\frac{\gamma L_{1}(A-B)\left\{4 \lambda[\lambda-1+(t-1)(1-\beta)]+(t-1)(t-2)(1-\beta)^{2}\right\}}{2(1+i \tan \alpha)[2 \lambda+(t-1)(1-\beta)]^{2}}
\end{gathered}
$$




$$
\left.\left.-\frac{\mu \gamma L_{1}(A-B)[3 \lambda+(t-1)(1-\beta)] \Theta_{3}}{\Theta_{2}^{2}(1+i \tan \alpha)[2 \lambda+(t-1)(1-\beta)]^{2}} \mid-2\right)\right] .
$$

Denoting:

$$
\begin{gathered}
Y:=\mid \frac{2 L_{2}}{L_{1}}-(B+1) L_{1} \\
-\frac{\gamma L_{1}(A-B)\left\{4 \lambda[\lambda-1+(t-1)(1-\beta)]+(t-1)(t-2)(1-\beta)^{2}\right\}}{2(1+i \tan \alpha)[2 \lambda+(t-1)(1-\beta)]^{2}} \\
-\frac{\mu \gamma L_{1}(A-B)[3 \lambda+(t-1)(1-\beta)] \Theta_{3}}{\Theta_{2}^{2}(1+i \tan \alpha)[2 \lambda+(t-1)(1-\beta)]^{2}} \mid
\end{gathered}
$$

if $Y \leq 2$, from (34) we obtain:

$$
\left|a_{3}-\mu a_{2}^{2}\right| \leq \frac{\left|L_{1}\right|(A-B)|\gamma|}{2[3 \lambda+(t-1)(1-\beta)]\left|\Theta_{3}\right| \sec \alpha} .
$$

Further, if $Y \geq 2$, from (34) we deduce:

$$
\begin{gathered}
\left|a_{3}-\mu a_{2}^{2}\right| \leq \frac{\left|L_{1}\right|(A-B)|\gamma|}{2[3 \lambda+(t-1)(1-\beta)]\left|\Theta_{3}\right| \sec \alpha}\left(\mid \frac{2 L_{2}}{L_{1}}-(B+1) L_{1}\right. \\
-\frac{\gamma L_{1}(A-B)\left\{4 \lambda[\lambda-1+(t-1)(1-\beta)]+(t-1)(t-2)(1-\beta)^{2}\right\}}{2(1+i \tan \alpha)[2 \lambda+(t-1)(1-\beta)]^{2}} \\
\left.-\frac{\mu \gamma L_{1}(A-B)[3 \lambda+(t-1)(1-\beta)] \Theta_{3}}{\Theta_{2}^{2}(1+i \tan \alpha)[2 \lambda+(t-1)(1-\beta)]^{2}} \mid\right) .
\end{gathered}
$$

An examination of the proof shows that the equality for (35) holds that if $p_{1}=0$, then $p_{2}=2$. Equivalently, by Lemma 3, we have $p\left(z^{2}\right)=p_{2}(z)=\frac{1+z^{2}}{1-z^{2}}$. Therefore, the extremal function of the class $\mathcal{P} \mathcal{S}_{\lambda}^{t}(\alpha, \beta ; \gamma ; \psi ; h ; A, B)$ is given by

$$
1+\frac{1+i \tan \alpha}{\gamma}\left[\frac{z^{1-t}\left[H^{\prime}(z)\right]^{\lambda}}{[(1-\beta) H(z)+\beta z]^{1-t}}-1\right]=\frac{(A+1) p\left(z^{2}\right)-(A-1)}{(B+1) p\left(z^{2}\right)-(B-1)} .
$$

Similarly, the equality for (36) holds that $p_{2}=2$. Equivalently, by Lemma 3, we have $p(z)=p_{1}(z)=\frac{1+z}{1-z}$. Therefore, the extremal function in $\mathcal{P} \mathcal{S}_{\lambda}^{t}(\alpha, \beta ; \gamma ; \psi ; h ; A, B)$ is given by

$$
1+\frac{1+i \tan \alpha}{\gamma}\left[\frac{z^{1-t}\left[H^{\prime}(z)\right]^{\lambda}}{[(1-\beta) H(z)+\beta z]^{1-t}}-1\right]=\frac{(A+1) p_{1}(z)-(A-1)}{(B+1) p_{1}(z)-(B-1)},
$$

and the proof of the theorem is complete.

Theorem 11. If $f(z)=z+a_{2} z^{2}+a_{3} z^{3}+\cdots \in \mathcal{S}_{\lambda}^{q}(t ; \alpha, \beta ; \gamma ; h, A, B)$, then for all $\mu \in \mathbb{C}$, we have:

$$
\left|a_{3}-\mu a_{2}^{2}\right| \leq \frac{(A-B)(1+q)|\gamma|}{2\left\{[3]_{q} \lambda+(t-1)(1-\beta)\right\}\left|\Theta_{3}\right| \sec \alpha} \max \{1 ;|2 \rho-1|\},
$$


where $\rho$ is given by

$$
\begin{gathered}
\rho:=\frac{1}{4}\left[B(1+q)+(3-q)+\frac{\mu \gamma(A-B)(1+q)\left\{[3]_{q} \lambda+(t-1)(1-\beta)\right\} \Theta_{3}}{(1+i \tan \alpha)\left\{[2]_{q} \lambda+(t-1)(1-\beta)\right\}^{2} \Theta_{2}^{2}}\right. \\
\left.+\frac{\gamma(1+q)(A-B)\left\{(1+q) \lambda[(1+q)(\lambda-1)+2(t-1)(1-\beta)]+(t-1)(t-2)(1-\beta)^{2}\right\}}{2(1+i \tan \alpha)\left\{[2]_{q} \lambda+(t-1)(1-\beta)\right\}^{2}}\right] .
\end{gathered}
$$

Proof. Using the definition of the quantum derivative, we can establish that:

$$
\begin{gathered}
\frac{1+i \tan \alpha}{\gamma}\left[\frac{z^{1-t}\left[\mathfrak{D}_{q} H(z)\right]^{\lambda}}{[(1-\beta) H(z)+\beta z]^{1-t}}-1\right]=\frac{1+i \tan \alpha}{\gamma}\left[\left\{[2]_{q} \lambda+(1-t)(1-\beta)\right\} \Theta_{2} a_{2} z\right. \\
+\left\{\left\{[3]_{q} \lambda+(t-1)(1-\beta)\right\} \Theta_{3} a_{3}+\frac{1}{2}[(1+q) \lambda[(1+q)(\lambda-1)+2(t-1)(1-\beta)]\right. \\
\left.\left.\left.+(t-1)(t-2)(1-\beta)^{2}\right] \Theta_{2}^{2} a_{2}^{2}\right\} z^{2}+\ldots\right] .
\end{gathered}
$$

Following the similar steps as in the proof of Theorem 4, we obtain:

$$
\begin{aligned}
& \frac{(A+1) w(z)+2+(A-1) q w(z)}{(B+1) w(z)+2+(B-1) q w(z)}-1=\frac{(1+q) p_{1}(A-B)}{4} z \\
& +\frac{(A-B)(1+q)}{4}\left[p_{2}-\frac{p_{1}^{2}}{4}\{B(1+q)+(3-q)\}\right] z^{2}+\ldots,
\end{aligned}
$$

and using (37) and (38) in (8), we obtain:

$$
a_{2}=\frac{\gamma(1+q) p_{1}(A-B)}{4(1+i \tan \alpha)\left\{[2]_{q} \lambda+(1-t)(1-\beta)\right\} \Theta_{2}},
$$

and:

$$
\begin{gathered}
a_{3}=\frac{(1+q)(A-B) \gamma}{4(1+i \tan \alpha)\left\{[3]_{q} \lambda+(t-1)(1-\beta)\right\} \Theta_{3}}\left[p_{2}-\frac{1}{4}(B(1+q)+(3-q)\right. \\
\left.\left.+\frac{\gamma(1+q)(A-B)\left\{(1+q) \lambda[(1+q)(\lambda-1)+2(t-1)(1-\beta)]+(t-1)(t-2)(1-\beta)^{2}\right\}}{2(1+i \tan \alpha)\left\{[2]_{q} \lambda+(t-1)(1-\beta)\right\}^{2}}\right) p_{1}^{2}\right] .
\end{gathered}
$$

From (39) and (40), we deduce that:

$$
\begin{gathered}
\left|a_{3}-\mu a_{2}^{2}\right|=\mid \frac{(1+q)(A-B) \gamma}{4(1+i \tan \alpha)\left\{[3]_{q} \lambda+(t-1)(1-\beta)\right\} \Theta_{3}}\left[p_{2}-\frac{1}{4}(B(1+q)+(3-q)\right. \\
+\frac{\mu \gamma(A-B)(1+q)\left\{[3]_{q} \lambda+(t-1)(1-\beta)\right\} \Theta_{3}}{(1+i \tan \alpha)\left\{[2]_{q} \lambda+(t-1)(1-\beta)\right\}^{2} \Theta_{2}^{2}} \\
\left.\left.+\frac{\gamma(1+q)(A-B)\left\{(1+q) \lambda[(1+q)(\lambda-1)+2(t-1)(1-\beta)]+(t-1)(t-2)(1-\beta)^{2}\right\}}{2(1+i \tan \alpha)\left\{[2]_{q} \lambda+(t-1)(1-\beta)\right\}^{2}}\right) p_{1}^{2}\right] \mid .
\end{gathered}
$$

Now, following the steps as in the proof of Theorem 10, we can establish the assertion of the theorem. 
Corollary 6 ([25] (Theorem 2.1)). If $f(z)=z+a_{2} z^{2}+a_{3} z^{3}+\cdots \in \mathcal{P} \mathcal{S}_{1}^{0}(\alpha, 0 ; \gamma ; \psi ; \mathfrak{h} ; 1,-1)$, where $\mathfrak{h}(z):=\frac{\sqrt{\pi z}}{2} \operatorname{erf}(\sqrt{z})$ and "erf" is the error function $\operatorname{erf}(z):=\frac{2}{\sqrt{\pi}} \int_{0}^{z} \exp \left(-t^{2}\right) d t$, then for all $\mu \in \mathbb{C}$ we have:

$$
\left|a_{3}-\mu a_{2}^{2}\right| \leq \frac{10|\gamma| L_{1}}{q(1+q) \sec \alpha} \max \left\{1 ;\left|\frac{L_{2}}{L_{1}}-\frac{9 \mu(q+1)+10}{10 q(1+i \tan \alpha)} \gamma L_{1}\right|\right\} .
$$

Proof. In Theorem 10 fixing $\beta=t=0, \lambda=A=1, B=-1$, and letting:

$$
H(z)=f(z) * \frac{\sqrt{\pi z}}{2} \operatorname{erf}(\sqrt{z})=z+\sum_{k=2}^{\infty} \frac{(-1)^{k-1}}{(2 k-1)(k-1) !} a_{k} z^{k}, z \in \mathbb{U},
$$

where "erf" is the error function given in the assumption (see [25]) and we obtain our result.

Letting $A=1, B=-1, t=\alpha=0, \lambda=1, h(z)=z+\sum_{k=2}^{\infty} \frac{(b)_{k-1}}{(c)_{k-1}} z^{k}, z \in \mathbb{U}, \gamma=1+0 i$ and $\psi(z)=z+\sqrt{1+z^{2}}$ in Theorem 10 we obtain the following special case:

Corollary 7 ([13] (Theorem 2)). If $f \in \mathcal{M L}_{b}^{c}\left(\beta ; z+\sqrt{1+z^{2}}\right)$ (see Remark 3 (ii)) is of the form (1), then for all $\mu \in \mathbb{C}$, we have:

$$
\left|a_{3}-\mu a_{2}^{2}\right| \leq\left|\frac{(c)_{2}}{(b)_{2}}\right| \frac{1}{2+\beta} \max \left\{1 ; \frac{|(\beta-3)(1+\beta) b(c+1)+2 \mu(2+\beta) c(b+1)|}{2(1+\beta)^{2}|b(c+1)|}\right\} .
$$

The inequality is sharp for each $\mu \in \mathbb{C}$.

For $b=c$, the above Corollary reduces to the next special case:

Example 6 ([13] (Corollary 1)). If $f \in \mathcal{M L}\left(\beta ; z+\sqrt{1+z^{2}}\right):=\mathcal{M L}_{b}^{b}\left(\beta ; z+\sqrt{1+z^{2}}\right)$ is of the form (1), then for all $\mu \in \mathbb{C}$ we have:

$$
\left|a_{3}-\mu a_{2}^{2}\right| \leq \frac{1}{2+\beta} \max \left\{1 ; \frac{|(\beta-3)(1+\beta)+2 \mu(2+\beta)|}{2(1+\beta)^{2}}\right\} .
$$

The inequality is sharp for each $\mu \in \mathbb{C}$.

For $\psi=\mathcal{T}$, where the function $\mathcal{T}$ is given by (24), we have $\Theta_{k}=\lambda=\gamma=1$. For $\alpha=\beta=t=0$ and $A=1, B=-1$, Theorem 10 reduces to the next result:

Corollary 8 ([18] (Theorem 5)). Let $0 \leq \eta<1<\vartheta$ and let the function $f \in \mathcal{S}(\eta, \vartheta)$. Then, for all $\mu \in \mathbb{C}$, we have

$$
\begin{gathered}
\left|a_{3}-\mu a_{2}^{2}\right| \leq \frac{\vartheta-\eta}{\pi} \sin \frac{\pi(1-\eta)}{\vartheta-\eta} \\
\cdot \max \left\{1 ;\left|\frac{1}{2}+(1-2 \mu) \frac{\vartheta-\eta}{\pi} i+\left(\frac{1}{2}-(1-2 \mu) \frac{\vartheta-\eta}{\pi} i\right) e^{2 \pi i \frac{1-\eta}{\vartheta-\eta}}\right|\right\} .
\end{gathered}
$$

Corollary 9 ([26] (Theorem 3.1)). If $f(z)=z+a_{2} z^{2}+a_{3} z^{3}+\cdots \in \mathcal{S}^{*}(\psi)$ and $\psi(z)=1+L_{1} z+L_{2} z^{2}+\ldots$, with $L_{1}, L_{2} \in \mathbb{R}, L_{1}>0$, then for all $\mu \in \mathbb{C}$ we have:

$$
\left|a_{3}-\mu a_{2}^{2}\right| \leq \frac{L_{1}}{2} \max \left\{1 ;\left|L_{1}+\frac{L_{2}}{L_{1}}-2 \mu L_{1}\right|\right\} .
$$


The inequality is sharp for the function $f_{*}$ given by

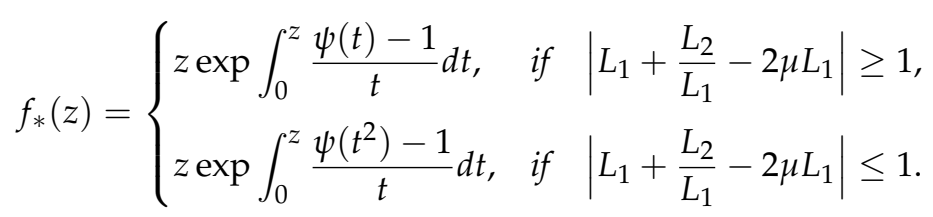

Proof. In Theorem 10, taking $A=1, B=-1, \Theta_{k}=\lambda=\gamma=1$ and $\alpha=\beta=t=0$ we obtain the inequality:

$$
\left|a_{3}-\mu a_{2}^{2}\right| \leq \begin{cases}\frac{L_{1}}{2}, & \text { if } \quad\left|L_{1}+\frac{L_{2}}{L_{1}}-2 \mu L_{1}\right| \leq 1 \\ \frac{L_{1}}{2}\left|L_{1}+\frac{L_{2}}{L_{1}}-2 \mu L_{1}\right|, & \text { if } \quad\left|L_{1}+\frac{L_{2}}{L_{1}}-2 \mu L_{1}\right| \geq 1\end{cases}
$$

Examining the last part in the proof of the Theorem 10, it follows that the first equality holds if $p_{1}=0, p_{2}=2$. Equivalently, by Lemma 3, we have $p(z)=p_{2}(z)=\frac{1+z^{2}}{1-z^{2}}$. Therefore, the extremal function of $\mathcal{S}^{*}(\psi)$ is given by

$$
\frac{z f^{\prime}(z)}{f(z)}=\psi\left(\frac{p_{2}(z)-1}{p_{2}(z)+1}\right)=\psi\left(z^{2}\right) .
$$

Similarly, the second equality holds if $p_{1}=2$. Equivalently, by Lemma 3, we have $p(z)=p_{1}(z)=\frac{1+z}{1-z}$. Therefore, the extremal function of $\mathcal{S}^{*}(\psi)$ is defined by

$$
\frac{z f^{\prime}(z)}{f(z)}=\psi\left(\frac{p_{1}(z)-1}{p_{1}(z)+1}\right)=\psi(z) .
$$

Finally, following a similar technique to that for the sharpness of Theorem 3.1 of [26], from the relations (42) and (43), we obtain (41).

\section{Conclusions}

By defining $\lambda$-pseudo-Bazilevič functions of complex order using subordination and Hadamard product, we were able to unify and extend the various classes of analytic function, and new extensions were discussed in detail. Furthermore, by replacing the ordinary differentiation with quantum differentiation, we attempted the discretization of some well-known results. Our main results have many applications, which here we only pointed out a few.

Author Contributions: Conceptualization, K.R.K., G.M. and T.B.; methodology, K.R.K., G.M. and T.B.; software, K.R.K., G.M. and T.B.; validation, K.R.K., G.M. and T.B.; formal analysis, K.R.K., G.M. and T.B.; investigation, K.R.K., G.M. and T.B.; resources, K.R.K., G.M. and T.B.; data curation, K.R.K., G.M. and T.B.; writing—original draft preparation, K.R.K. and G.M.; writing-review and editing, K.R.K., G.M. and T.B.; visualization, K.R.K., G.M. and T.B.; supervision, K.R.K., G.M. and T.B.; project administration, K.R.K., G.M. and T.B. All authors have read and agreed to the published version of the manuscript.

Funding: This research received no external funding.

Institutional Review Board Statement: Not applicable.

Informed Consent Statement: Not applicable.

Data Availability Statement: Not applicable.

Acknowledgments: The authors are grateful to the reviewer for the valuable remarks, comments, and advices that help us to remove some mistakes that appeared in the manuscript and to improve the quality of the paper. 
Conflicts of Interest: The authors declare no conflict of interest.

\section{References}

1. Bazilevič, I.E. On a case of integrability in quadratures of the Loewner-Kufarev equation. Matematicheskii Sbornik 1955, 79, 471-476.

2. Babalola, K.O. On $\lambda$-pseudo-starlike functions. J. Class. Anal. 2013, 3, 137-147. [CrossRef]

3. Kuroki, K.; Owa, S. Notes on new class for certain analytic functions. RIMS Kokyuroku 2011, 1772, $21-25$.

4. Annaby, M.H.; Mansour, Z.S. Q-Fractional Calculus and Equations; Lecture Notes in Mathematics, 2056; Springer: Berlin/Heidelberg, Germany, 2012.

5. Aral, A.; Gupta, V.; Agarwal, R.P. Applications of Q-Calculus in Operator Theory; Springer: New York, NY, USA, 2013.

6. Bulboacă, T. Differential Subordinations and Superordinations. Recent Results; House of Science Book Publisher: Cluj-Napoca, Romania, 2005.

7. Pommerenke, C. Univalent Functions; Vandenhoeck \& Ruprecht: Göttingen, Germany, 1975.

8. Ma, W.C.; Minda, D. A unified treatment of some special classes of univalent functions. In Proceedings of the Conference on Complex Analysis, Tianjin, China, 19-23 June 1992; International Press: Cambridge, MA, USA, 1992; pp. 157-169.

9. Karthikeyan, K.R.; Murugusundaramoorthy, G.; Nistor-Şerban, A.; Răducanu, D. Coefficient estimates for certain subclasses of starlike functions of complex order associated with a hyperbolic domain. Bull. Transilv. Univ. Braşov Ser. III 2020, 13, 595-610.

10. Raina, R.K.; Sokół, J. On a class of analytic functions governed by subordination. Acta Univ. Sapientiae Math. 2019, 11, 144-155. [CrossRef]

11. Sokół, J.; Thomas, D.K. Further results on a class of starlike functions related to the Bernoulli lemniscate. Houst. J. Math. 2018, 44, 83-95.

12. Malik, S.N.; Mahmood, S.; Raza, M.; Farman, S.; Zainab, S. Coefficient inequalities of functions associated with petal type domains. Mathematics 2018, 6, 298. [CrossRef]

13. Murugusundaramoorthy, G; Bulboacă, T. Hankel determinants for new subclasses of analytic functions related to a shell shaped region. Mathematics 2020, 8, 1041. [CrossRef]

14. Goodman, A.W. Univalent Functions; Mariner Publishing Co., Inc.: Tampa, FL, USA, 1983; Volume II.

15. Rogosinski, W. On the coefficients of subordinate functions. Proc. Lond. Math. Soc. 1943, 48, 48-82. [CrossRef]

16. Xu, N.; Yang, D. Some criteria for starlikeness and strongly starlikeness. Bull. Korean Math. Soc. 2005, 42, 579-590. [CrossRef]

17. Mocanu, P.T.; Oros, G.; Sufficient conditions for starlikeness. II. Stud. Univ. Babeş-Bolyai Math. 1998, 43, 49-53.

18. Sim, Y.J.; Kwon, O.S. Notes on analytic functions with a bounded positive real part. J. Inequal. Appl. 2013, 370, 1-9. [CrossRef]

19. Khan, B; Srivastava, H.M.; Khan, N.; Darus, M.; Tahir, M.; Ahmad, Q.Z. Coefficient estimates for a subclass of analytic functions associated with a certain leaf-like domain. Mathematics 2020, 8, 1334. [CrossRef]

20. Kaplan, W. Advanced Calculus, 4th ed.; Addison Wesley: Reading, MA, USA, 1991.

21. Selvaraj, C.; Karthikeyan, K.R. Certain classes of analytic functions of complex order involving a family of generalized differential operators. J. Math. Inequal. 2008, 2, 449-458. [CrossRef]

22. Xu, Q.-H.; Lv, C.-B.; Luo, N.-C.; Srivastava, H.M. Sharp coefficient estimates for a certain general class of spirallike functions by means of differential subordination. Filomat 2013, 27, 1351-1356. [CrossRef]

23. Libera, R.L. Univalent $\alpha$-spiral functions. Can. J. Math. 1967, 19, 449-456. [CrossRef]

24. Karthikeyan, K.R.; Selvam, A.; Sooriya Kala, P. On a class of analytic function defined using differential operator. Acta Univ. Apulensis Math. Inform. 2012, 31, 163-178.

25. Ramachandran, C.; Vanitha, L.; Kanas, S. Certain results on $q$-starlike and $q$-convex error functions. Math. Slovaca 2018, 68, 361-368. [CrossRef]

26. Tu, Z.; Xiong, L. Unified solution of Fekete-Szegő problem for subclasses of starlike mappings in several complex variables. Math. Slovaca 2019, 69, 843-856. [CrossRef] 